\title{
Genetic variations in the Wnt signaling pathway affect lung function in asthma patients
}

\author{
S.-H. Wang ${ }^{1,2}$, F. Xu ${ }^{1}$, H.-X. Dang ${ }^{1}$ and L. Yang $^{1}$ \\ ${ }^{1}$ Pediatric Intensive Care Unit, Children's Hospital, \\ Chongqing Medical University, Chongqing, China \\ ${ }^{2}$ Women and Children Health Institute, Futian, Shenzhen, Guangdong, China \\ Corresponding author: F. Xu \\ E-mail: wangshaohuaxf@yeah.net
}

Genet. Mol. Res. 12 (2): 1829-1833 (2013)

Received February 6, 2012

Accepted August 24, 2012

Published January 4, 2013

DOI http://dx.doi.org/10.4238/2013.January.4.1

\begin{abstract}
Identifying genetic determinants for lung function is important in providing insight into the pathophysiology of asthma. The Wnt signal pathway plays a role in lung development and in asthma pathogenesis. However, whether genetic polymorphisms of Wnt signal pathway are associated with lung function in asthma patients remain unclear. We genotyped 2 single nucleotide polymorphisms (SNPs, rs2929973 and rs6581612) involved in the Wnt signal pathway in a cohort of 560 Chinese Han asthmatic children. Associations between each SNP and lung function, in a baseline exam, were tested using multiple linear regression models. We found that rs2929973 of the WISP1 gene was significantly associated with forced expiratory volume in $1 \mathrm{~s}$ (FEV1), with the G allele conferring significantly lower FEV1 values. However, the rs6581612 SNP of the WIF1 gene was not associated with differences in FEV1 values. We conclude that genetic variants in Wnt are associated with lung function and suggest that Wnt participates in inflammatory pathways that have an impact on the level of lung function.
\end{abstract}

Key words: Wnt signaling pathway; Lung function; Asthma 


\section{INTRODUCTION}

Pulmonary function is among the most important health indicators, being a strong predictor of long-term morbidity and mortality and a marker of disease severity in respiratory tract diseases (Tockman and Comstock, 1989). Heritability estimates of lung function in the general population suggest that genetic factors explain 25 to $37 \%$ of between-subject variability in forced expiratory volume in $1 \mathrm{~s}$ (FEV1), forced vital capacity, and the ratio of FEV1/ forced vital capacity (Redline et al., 1989; Coultas et al., 1991; Wilk et al., 2000; Joost et al., 2002). Twin studies in subjects with asthma have suggested that within this susceptible population, genetic factors may explain a large proportion of altered lung function (77-91\% for FEV1) even after accounting for smoking and the shared genetic effects of body size (Hankins et al., 1982; Hubert et al., 1982).

Evidence has suggested that the study of molecular pathways that regulate lung development may provide important insights on the pathogenesis of pulmonary disease. The early stages of lung development are clearly dependent on complex molecular pathways, and studies have suggested that these molecular pathways can be recapitulated in postnatal life and cause pathologic changes in complex respiratory diseases (Gross and Hunninghake, 2001; Selman et al., 2008).

The number of studies investigating Wnt signaling in the lungs has increased substantially in recent years. The first evidence of Wnt expression in the lung was presented by Selman et al. (2008). More recently, the role of Wnt signaling in lung development and function has emerged as a promising research area. Sharma et al. (2010) have suggested that the genes involved in the Wnt signaling pathway, such as the rs2929973 of the Wnt-1-induced secreted protein 1 (WISP1) gene and rs6581612 of the Wnt-inhibitory factor 1 (WIF1) gene, may be associated with lung function in asthma. However, no replicated studies have been reported in other ethnic populations to date. In the present study, we aimed to analyze the relationships between genetic polymorphism in the Wnt signal pathway (rs2929973 and rs6581612) and lung function in the Chinese population.

\section{MATERIAL AND METHODS}

\section{Population}

This study was approved by the Local Ethics Committee. The children who participated provided oral assent, and their parents provided written informed consent. The study was performed at the Children's Hospital of Chongqing Medical University and included 650 children aged 5 to 12 years with mild to moderate asthma who were examined from January 2006 to December 2010. Entry criteria included asthma symptoms or medication use for $\geq 6$ months during the previous year and airway responsiveness with a provocative concentration of methacholine causing a $20 \%$ reduction in FEV1 of $\leq 12.5 \mathrm{mg} / \mathrm{mL}$.

\section{Phenotypes}

The primary phenotype of interest in the cohorts was baseline, pre-bronchodilator FEV1 as a percentage of predicted. Baseline spirometry was performed at randomization after a 28-day period during which only as-needed albuterol was allowed. 


\section{DNA sample extraction}

Blood samples were collected with a standard venipuncture technique and tubes containing ethylenediaminetetraacetic acid. DNA was extracted from peripheral vein blood leukocytes using a whole-blood genome extraction kit (Beijing Bioteke Corporation, Beijing, China) according to manufacturer instructions. Samples were coded to protect donor identity and allow blinding of the investigators who carried out the genotyping.

\section{Genotyping}

We selected single nucleotide polymorphisms (SNPs) according to a protocol described by Sharma et al. (2010). We chose rs2929973 of the WISP1 gene and rs6581612 of the WIF1 gene. Quantification of DNA extracted was performed using a NanoDrop ND-1000 spectrophotometer (NanoDrop Technologies, Wilmington, DE, USA). Genotyping was performed using the TaqMan ${ }^{\circledR}$ SNP Genotyping Assay (Applied Biosystems Inc., CA, USA). To ensure verified results, we used sequenced genomic DNAs as positive controls in our assays.

\section{Statistical analysis}

Data analysis was performed using SPSS for Windows (version 13.0, SPSS Institute, Chicago, IL, USA). To determine whether the polymorphisms were in Hardy-Weinberg equilibrium, the actual and predicted genotype counts for controls were compared using chi-square analysis with 2 degrees of freedom. Univariate associations between SNPs and the phenotype of interest (FEV1 as a percentage of predicted) were tested using univariate linear regression. Multivariate linear regression models were used to control for potential confounders. In these models, the genotype for each SNP was coded as a 3-level categorical variable (additive genetic model) or as a dummy-coded variable.

\section{RESULTS}

\section{Characteristics of study participants}

The study cohort consisted of 650 subjects. The clinical characteristics of the study population are shown in Table 1.

\begin{tabular}{lc} 
Table 1. Characteristics of study participants. \\
\hline Risk factor & No. (\%) or mean (SD) \\
\hline Age [mean (SD)] & $8.3(2.4)$ \\
Gender, male [N (\%)] & $378(58.2)$ \\
FEV1 (\% predicted) [mean (SD)] & $93.3(14.7)$ \\
Eosinophil count [geometric mean (SD)] & $5.87(2.12)$ \\
IgE [geometric mean (SD)] & $406.5(5.2)$ \\
Paternal asthma [N (\%)] & $121(18.6)$ \\
Maternal asthma [N (\%)] & $162(24.9)$ \\
Maternal cigarette smoking during pregnancy [N (\%)] & $90(13.8)$ \\
\hline SD standar deviation.
\end{tabular}

$\mathrm{SD}=$ standard deviation. 


\section{Genotype and allele frequencies}

All genotyped SNPs were in Hardy-Weinberg equilibrium. Table 2 shows detailed information for each SNP as well as the allele frequencies.

Table 2. Genotype and allele frequencies.
\begin{tabular}{lcccccc}
\multicolumn{1}{l}{ SNPs } & $\mathrm{N}$ & Genotype (\%) & \multicolumn{2}{c}{ Allele (frequency) } \\
\hline rs2929973 & 560 & $\mathrm{GG}$ & $\mathrm{GT}$ & $\mathrm{TT}$ & $\mathrm{G}$ & $\mathrm{T}$ \\
& & $58(10.4)$ & $302(53.9)$ & $200(35.7)$ & 0.38 & 0.62 \\
rs6581612 & 560 & $\mathrm{AA}$ & $\mathrm{AC}$ & $\mathrm{CC}$ & $\mathrm{A}$ & $\mathrm{C}$ \\
& & $545(97.3)$ & $14(2.5)$ & $1(0.2)$ & 0.99 & 0.01 \\
\hline
\end{tabular}

SNPs $=$ single nucleotide polymorphisms.

\section{Associations with baseline FEV1}

Using general linear model analysis, we found that rs2929973 was significantly associated with baseline FEV1 levels in dominant, recessive, and additive models (Table 3).

\begin{tabular}{|c|c|c|c|c|c|c|c|}
\hline \multirow[t]{2}{*}{$\overline{\mathrm{SNP}}$} & \multirow[t]{2}{*}{ Wild/rare allele } & \multicolumn{3}{|c|}{ FEV1 (\% predicted), mean (SD) } & \multicolumn{3}{|c|}{ Model } \\
\hline & & Homozygous for rare allele & Heterozygous & Homozygous for wild allele & $\mathrm{P}(\operatorname{Rec})$ & P (Dom) & P (Add) \\
\hline rs2929973 & $\mathrm{G} / \mathrm{T}$ & $98.3(14.2)$ & $89.5(13.2)$ & $76.4(13.5)$ & $<0.01$ & $<0.01$ & $<0.01$ \\
\hline rs6581612 & $\mathrm{A} / \mathrm{C}$ & $94.6(13.6)$ & 93.1 (14.6) & $90.7(14.1)$ & 0.765 & 0.354 & 0.137 \\
\hline
\end{tabular}

$\mathrm{SNP}=$ single nucleotide polymorphism; $\mathrm{SD}=$ standard deviation; $\mathrm{Rec}=$ recessive; Dom $=$ dominant; Add $=$ additive.

\section{DISCUSSION}

We found that variation in Wnt pathway genes is associated with lung function in children with asthma. This study is the first to investigate common allelic variants in the Wnt pathway gene and their association with lung function in Han Chinese children.

The results of a previous study (Sharma et al., 2010) have indicated that the involvement of WISP1 and WIF1 in the Wnt signal pathway is associated with both intrauterine airway development and lung function impairment in susceptible populations. Colston et al. (2007) have demonstrated a role for WISP1 in postinfarction cardiac remodeling by demonstrating the proliferative effect of WISP1 on fibroblasts. WIF1 inhibits Wnt signaling by directly binding to Wnt ligands, thus disrupting their ability to bind to the Wnt/frizzled receptor (Van Scoyk et al., 2008). Decreased WIF1 expression in human disease states, including lung cancer, has been related to hypermethylation of the promoter region of the gene (Ai et al., 2006). Sun et al. (2008) have previously shown that abrogation of activin-like kinase 3-mediated bone morphogenetic protein (BMP) signaling in lung epithelial cells during early lung development disrupts cell differentiation and proliferation, resulting in abnormal lung branching morphogenesis. The murine model used by these authors demonstrates that changes in BMP 
signaling subsequently cause respiratory distress syndrome in the early postnatal period. Analysis of the lung tissue of activin-like kinase 3 conditional knockout mice has demonstrated that decreased BMP signaling is associated with increased canonical Wnt signaling, as evidenced by increased phosphorylation of the Wnt coreceptor low-density lipoprotein receptor-related protein 6 and activation of downstream $\beta$-catenin (Sun et al., 2008).

In the present study, we selected 2 SNPs (rs2929973 and rs6581612) of the WIF1 and WISP1 genes and found that rs2929973 was associated with lung function in asthmatic children. However, rs6581612 was not associated with lung function in this population.

\section{ACKNOWLEDGMENTS}

We thank all the participants for the donation of blood and DNA.

\section{REFERENCES}

Ai L, Kim WJ, Kim TY, Fields CR, et al. (2006). Epigenetic silencing of the tumor suppressor cystatin M occurs during breast cancer progression. Cancer Res. 66: 7899-7909.

Colston JT, de la Rosa SD, Koehler M, Gonzales K, et al. (2007). Wnt-induced secreted protein-1 is a prohypertrophic and profibrotic growth factor. Am. J. Physiol. Heart Circ. Physiol. 293: H1839-H1846.

Coultas DB, Hanis CL, Howard CA, Skipper BJ, et al. (1991). Heritability of ventilatory function in smoking and nonsmoking New Mexico Hispanics. Am. Rev. Respir. Dis. 144: 770-775.

Gross TJ and Hunninghake GW (2001). Idiopathic pulmonary fibrosis. N. Engl. J. Med. 345: 517-525.

Hankins D, Drage C, Zamel N and Kronenberg R (1982). Pulmonary function in identical twins raised apart. Am. Rev. Respir. Dis. 125: 119-121.

Hubert HB, Fabsitz RR, Feinleib M and Gwinn C (1982). Genetic and environmental influences on pulmonary function in adult twins. Am. Rev. Respir. Dis. 125: 409-415.

Joost O, Wilk JB, Cupples LA, Harmon M, et al. (2002). Genetic loci influencing lung function: a genome-wide scan in the Framingham Study. Am. J. Respir. Crit. Care Med. 165: 795-799.

Redline S, Tishler PV, Rosner B, Lewitter FI, et al. (1989). Genotypic and phenotypic similarities in pulmonary function among family members of adult monozygotic and dizygotic twins. Am. J. Epidemiol. 129: 827-836.

Selman M, Pardo A and Kaminski N (2008). Idiopathic pulmonary fibrosis: aberrant recapitulation of developmental programs? PLoS Med. 5: e62.

Sharma S, Tantisira K, Carey V, Murphy AJ, et al. (2010). A role for Wnt signaling genes in the pathogenesis of impaired lung function in asthma. Am. J. Respir. Crit. Care Med. 181: 328-336.

Sun J, Chen H, Chen C, Whitsett JA, et al. (2008). Prenatal lung epithelial cell-specific abrogation of Alk3-bone morphogenetic protein signaling causes neonatal respiratory distress by disrupting distal airway formation. Am. $J$. Pathol. 172: 571-582.

Tockman MS and Comstock GW (1989). Respiratory risk factors and mortality: longitudinal studies in Washington County, Maryland. Am. Rev. Respir. Dis. 140: S56-S63.

Van Scoyk M, Randall J, Sergew A, Williams LM, et al. (2008). Wnt signaling pathway and lung disease. Transl. Res. 151: 175-180.

Wilk JB, Djousse L, Arnett DK, Rich SS, et al. (2000). Evidence for major genes influencing pulmonary function in the NHLBI family heart study. Genet. Epidemiol. 19: 81-94. 